\title{
Clinician-Targeted Intervention and Patient-Reported Counseling on Physical Activity
}

Jennifer K. Carroll, MD, MPH; Paul C. Winters, MS; Mechelle R. Sanders; Francesca Decker, MD, MPH; Thanh Ngo; Christopher N. Sciamanna, MD, MPH

Suggested citation for this article: Carroll JK, Winters PC, Sanders MR, Decker F, Ngo T, Sciamanna CN. ClinicianTargeted Intervention and Patient-Reported Counseling on Physical Activity. Prev Chronic Dis 2014;11:130302. DOI: http://dx.doi.org/10.5888/pcd11.130302 国.

PEER REVIEWED

\section{Abstract}

\section{Introduction}

Limited time and lack of knowledge are barriers to physical activity counseling in primary care. The objective of this study was to examine the effectiveness of a clinician-targeted intervention that used the 5As (Ask, Advise, Agree, Assist, Arrange) approach to physical activity counseling in a medically underserved patient population.

\section{Methods}

Family medicine clinicians at 2 community health centers were randomized to Group 1 or Group 2 intervention. Both clinician groups participated in 4 training sessions on the 5 As for physical activity counseling; Group 2 training took place 8 months after Group 1 training. Both groups were trained to refer patients to a community exercise program. We used a pre-post analysis to evaluate the effectiveness of the intervention on clinician use of 5As. Eligible patients $(\mathrm{n}=319)$ rated their clinicians' counseling skills by using a modified Physical Activity Exit Interview (PAEI) survey. Clinicians $(n=10)$ self-assessed their use of the 5 As through a survey and interviews.

\section{Results}

Both patient and clinician groups had similar sociodemographic characteristics. The PAEI score for both groups combined increased from 6.9 to 8.6 (on a scale of $0-15)$ from baseline to immediately postintervention $(P=.01)$ and was $8.2(P=.09)$ at 6 -month follow-up; most of the improvement in PAEI score was due to increased use of 5 As skills by Group 2 clinicians. Group 1 reported difficulty with problem solving, whereas Group 2 reported ease of referral to the community exercise program.

\section{Conclusion}

A clinician training intervention showed mixed results for 5 As physical activity counseling.

\section{Introduction}

Americans made 560 million visits to primary care physicians in 2010 (1). Most visits were for prevention and treatment of chronic conditions for which physical activity counseling would be appropriate. Even modestly effective evidence-based physical activity interventions in primary care settings could have powerful health benefits for patients (2).

Yet clinicians face significant barriers to implementing evidence-based interventions, especially for underserved populations that have limited resources and competing demands (3). The implications of clinicians not using evidencebased interventions include worsening health disparities related to inadequate physical activity.

Some barriers to implementing evidence-based interventions could be addressed by the " 5 As," an evidence-based clinical counseling framework in which clinicians are encouraged to Ask about (or Assess), Advise on, Agree upon, Assist with, and Arrange follow-up on patients' behavior-change efforts (4-11). The 5As can be used briefly and during 
multiple visits (12) and have been endorsed by the US Preventive Services Task Force (13) and others (14-16) and more recently for obesity counseling $(17,18)$.

Despite recommendations to use the $5 \mathrm{As}$, evidence for the feasibility of implementing the $5 \mathrm{As}$ in "real-world" settings is limited. In 2010, the US Preventive Services Task Force updated its recommendations on physical activity counseling (19), concluding that the evidence for low-intensity (30 minutes or less) counseling interventions was mixed and that further study is needed to evaluate them. Low-intensity counseling interventions are especially important because they are more likely than high-intensity interventions to be implemented in primary care settings.

The objective of this study was to examine the effectiveness of a clinician-targeted intervention that used the 5 As approach to physical activity counseling in a medically underserved patient population.

\section{Methods}

The study design and protocol are described in detail elsewhere (20). Briefly, the study used a 2-group pragmatic pilot randomized-controlled-trial design; 13 clinicians were randomized to receive the intervention; patients $(n=319)$ were the primary unit of analysis. For practical and logistical reasons, the clinicians were randomized so that Group 1 clinicians $(n=6)$ participated in the intervention first; Group $2(n=7)$ participated in the intervention 8 months later. The intervention consisted of a clinician training program designed to increase use of the 5As for physical activity counseling with patients. Outcomes were patient and clinician assessments of the effectiveness of the intervention in increasing use of the $5 \mathrm{As}$ for physical activity counseling. Other than the timing, there was no difference in the intervention or the assessment for the 2 groups. The University of Rochester Review Board approved the study protocol. The intervention period started in June 2009 and ended with the final follow-up data collection in October 2011.

\section{Recruitment, enrollment, randomization of clinicians and patients}

Clinicians and patients were recruited through 2 federally qualified health centers in Rochester, New York, serving a predominantly low-income, racially/ethnically diverse population of 14,000 patients. Details on inclusion and exclusion criteria for participants are described elsewhere (20). Clinicians were eligible if they practiced at one of the aforementioned centers. Clinicians were recruited, enrolled, and randomized to Group 1 or 2 from January through March 2009 before patient recruitment began (20). Of 15 clinicians eligible, 13 consented to participate (9 family physicians, 2 family nurse practitioners, and 2 physician assistants) and were randomized. Of the 13 clinicians randomized, 2 clinicians relocated and one retired, leaving 10 for analysis ( 5 in Group 1 and 5 in Group 2). Neither clinicians nor research staff was blinded to the assignment of clinicians. Clinicians were paid up to $\$ 365$ for participation in all study activities.

Patients were eligible if they were currently enrolled at one of the health centers, were aged 18 years or older, were able to provide informed consent, and made a health maintenance visit or follow-up visit for a chronic condition for which physical activity counseling would be appropriate. Nurse assistants mentioned the study to the patients when they were in the examination room. If the patient expressed interest, a research assistant entered the room to provide additional details about the study; patients who agreed to participate signed an informed consent form. For ethical and regulatory reasons, patients were not blinded to study topic and objective; as part of the informed consent process, they were made aware of these. However, they were blinded to time point (baseline, postintervention, 6-month followup) and did not know whether or when their clinicians took part in the intervention; patient participants were paid \$20 for participation.

During the study period, 1,029 patient participants were assessed for eligibility. Of these, 481 (or $46.7 \%$ of those assessed) were ineligible. Among the remaining 548 patients, 325 consented to participate (Figure). Of the 325 patients, 319 completed surveys for analysis. 


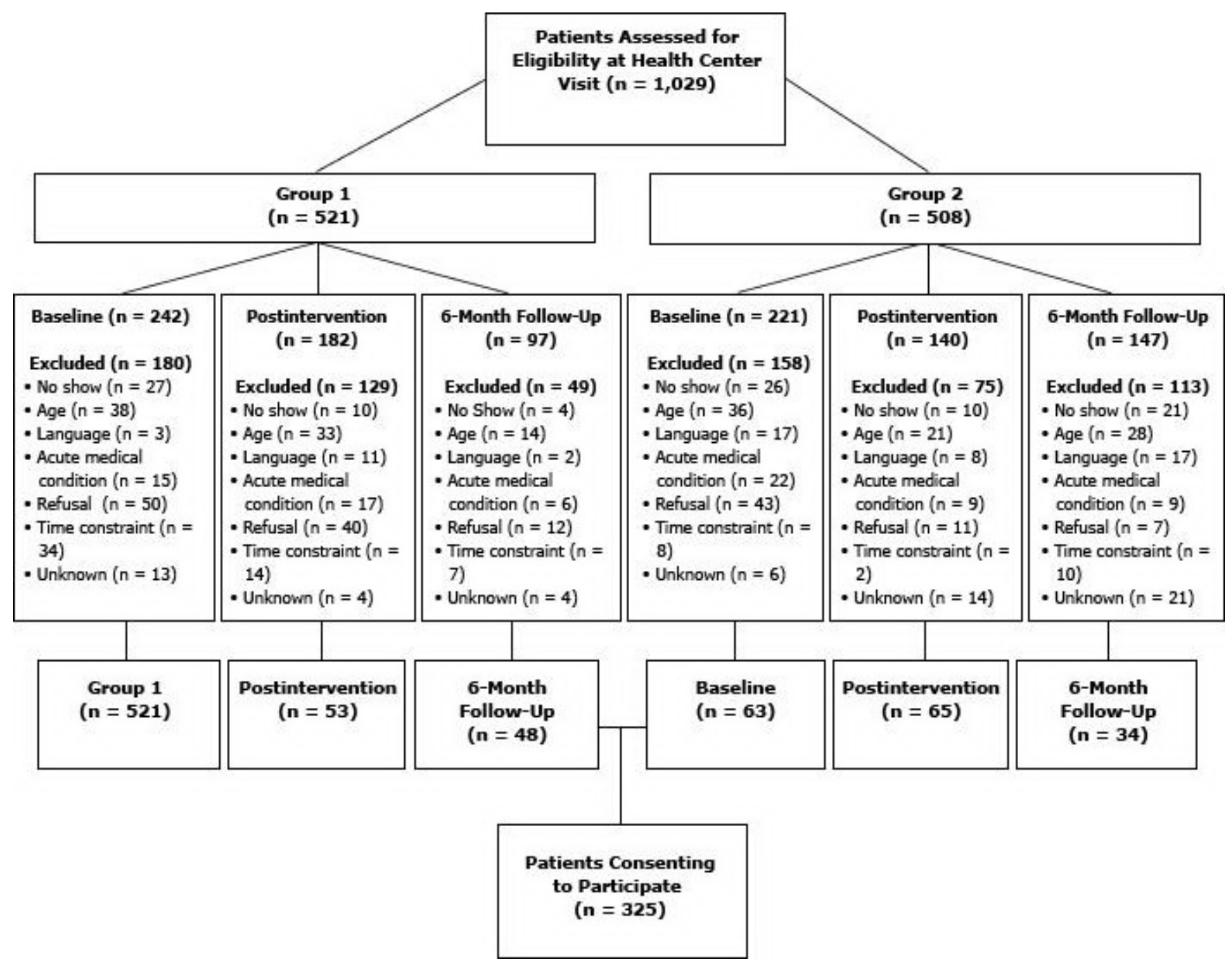

Figure. Assessment of patient participant eligibility and reasons for exclusions from study. Within each group, patients at baseline, postintervention, and 6-month follow-up were assessed independently of one another (ie, patients participating at baseline did not necessarily participate at postintervention or 6-months postintervention). [A text description of this figure is also available.]

\section{Intervention}

The intervention consisted of 4 one-hour training sessions for clinicians at the health center sites (20). Clinicians were trained through techniques known to be effective for communication training (21-23): didactic materials, skills and competency checklists, role-play, and cognitive rehearsal. Training topics consisted of introduction to the 5As, current recommended guidelines for physical activity, techniques to elicit patient motivation, strategies to problem-solve, tips on documentation in electronic health records, and a list of community resources for physical activity. As part of the Assist and Arrange steps, clinicians were taught to refer patients to a community exercise program (the Healthy Living Program), which had partnered with the federally qualified health center. The training period was interactive, and at its conclusion, each clinician received a competency checklist noting their accomplishment of the 5 As completed by a standardized patient (a person who realistically portrays a patient with a certain health condition). No booster sessions or other follow-up training activities were provided. All training sessions were audio-recorded. Two students listened to the recordings and completed a checklist of predefined training goals and activities to ensure fidelity.

\section{Patient-reported outcome measures}

For each clinician, patients completed a survey at 3 points: baseline, immediately postintervention, and at 6-month follow-up. Each patient completed 1 survey, so different groups of patients provided surveys at each point. Patients completed the survey, a modified version of the Physical Activity Exit Interview (PAEI) (24), after their office visit. The PAEI is a validated 12-item measure asking patients to report clinician use of 5 As for physical activity counseling. Patients dichotomously (yes/no) answered questions such as, "Did your doctor advise you to become more physically active?"; "Did your doctor discuss difficult situations you might encounter or problems you might have in trying to 
become more physically active?"; and "Did you and your doctor put the plan to become more physically active in writing?" The PAEI was modified for this intervention by adding 3 questions: "Has your provider ever asked you about your confidence to change your exercise habits?"; "Has your provider ever asked you about your willingness to change your exercise habits?"; and "Has your provider ever referred you to other programs, resources, consultants, etc., to help you with physical activity?” The score range for the modified PAEI was o to 15 (ie, 1 point for each yes). The patient survey also asked about sociodemographic characteristics, barriers to physical activity, and support and resources for physical activity.

\section{Clinician baseline and follow-up assessments}

Each clinician completed baseline and 6-month follow-up surveys. The surveys asked about demographic characteristics, current practice of physical activity counseling, confidence in using 5 As in counseling on physical activity, and knowledge of resources that could meet their patients' needs. Their confidence in using counseling skills was self-rated on a 5 -point Likert scale $(1=$ not confident, $5=$ very confident). At follow-up, each clinician also completed a 30-minute interview to provide feedback about the overall experience in the intervention, changes in skills, problems or difficulties, training techniques, satisfaction with the intervention, and suggestions for improvement.

\section{Statistical analysis}

All analyses were based on a significance level of .05. Two-sample $t$ tests for continuous variables and $\chi^{2}$ tests for categorical variables were used to evaluate the success of the randomization in balancing baseline covariates between groups. From the power and sample-size analysis, given our sample size, we could detect a difference of 1.5 or greater in PAEI scores between baseline and follow-up with $80 \%$ power. Data were analyzed with generalized estimating equations (GEE) models with patients nested within clinician using a negative-binomial link to account for the distribution of PAEI scores. We chose to use GEE models to account for nesting of patients within clinician and a possible unknown correlation between outcomes and to obtain robust standard errors. Also, GEE models provide a practical method for analyzing conditional responses that may depart from the normality assumption required for general linear models. Finally, we determined effect sizes and categorized them as small, medium, or large (25). For the qualitative analysis of interviews, we conducted interviews of patients and clinicians after the patient visit. All interviews were audio-recorded, transcribed, coded, and analyzed by using a constant comparative analysis technique.

\section{Results}

Three-quarters of clinicians were women; 66\% were white, 25\% African American, and 9\% Asian. The average age of clinicians was 49 years (range, 31-73 y), and the average length of practice was 15 years (range, 2-33 y). Group 1 and Group 2 patients did not differ significantly by any sociodemographic or health characteristic, so data for both groups were combined (Table 1). Patients' mean age was 43 years, and 69.9\% were African American. Most (61.4\%) had public insurance, and the mean body mass index (BMI) was 32.3. The most common weight-related conditions were hypertension (48.5\%), chronic pain (42.3\%), depression (32.4\%), and diabetes (21.3\%); 70\% of patients had more than 1 weight-related chronic condition, and $51 \%$ had 3 or more conditions.

In the mixed model controlling for clinician as a random effect, the PAEI score for both groups combined increased from 6.9 at baseline to 8.6 at postintervention $(P=.01)$ (Table 2). The medium effect size (o.34) was not sustained at 6 -month follow-up (mean PAEI score at 6 months, $8.2 ; P=.09$ ). The average PAEI score did not change significantly at either point for Group 1, whereas the average PAEI score increased at both points (from baseline) for Group 2 (Table 2). The PAEI score at baseline for Group 1 (7.5) was higher than the score at baseline for Group 2 (6.3) because Group 1 patients rated their clinicians higher on Assess and Assist items. However, at 6-month follow-up, Group 2 patients rated their clinicians higher on all PAEI items than did Group 1 patients, especially for Assess and Assist (Table 3). Of the 3 PAEI items added for this intervention, the scores for 2 items increased (Table 3).

From baseline to 6-month follow-up, clinician confidence increased significantly for assessing an exercise history, negotiating an exercise plan, turning setbacks into learning, helping patients cope with barriers, counseling in a costeffective way, and having knowledge of community resources that could meet patients' needs (Table 4).

\section{Qualitative feedback from clinicians}

From the clinician interviews, some differences emerged for Groups 1 and 2 in their perceptions of their 5 As counseling skills. Group 1 clinicians (whose score did not change) commented frequently on the challenges of problem solving with patients ("getting lost in the barriers" as stated by 1 clinician). Group 1 clinicians felt that the training was too brief and that booster trainings would have been helpful. They requested more feedback on their counseling skills (either from the research team or their peers) and support beyond the office visit to encourage patients and help with problem solving. In contrast, Group 2 clinicians (whose PAEI score was lower than Group 1's at baseline but increased at follow-up) mentioned the value and ease of referral to the community exercise program. The partnership for referral to the community exercise program was newly initiated when Group 1 took part in the study and was more established 
by the time Group 2 took part. Group 2 clinicians also commented that the 5 As framework was useful for raising awareness and reminding them of the importance of counseling on physical activity.

\section{Discussion}

This study evaluated the effect of a clinician-targeted intervention on patient- and clinician-reported use of the 5 As for physical activity counseling. The intervention used innovative, interactive clinician training techniques and focused exclusively on a medically underserved population not well represented in this type of research. The main finding was that the intervention increased patient-reported improvements in 5As counseling on physical activity immediately postintervention but not at 6-month follow-up, although this improvement was primarily due to higher scores for Group 2 clinicians. Group 1 clinicians had a higher baseline PAEI score, which may have limited the effectiveness of the intervention for them. Also, the community exercise program may have provided a greater incentive for Group 2 clinicians to use the 5 As because the program was better established for them than for Group 1 clinicians.

There are some practical lessons learned from this study. First, the development of the referral process for the community exercise program took time to function effectively, and this delay affected clinician behavior (ie, familiarity with and ease of referral to the program). Research on the effectiveness of community exercise programs for clinician referrals is lacking; others have commented on the potential demand and the difficulty of establishing effective partnerships for referral. A recent meta-analysis of primary care-based physical activity counseling (26) and recent US Preventive Services Task Force recommendations (27) identified evaluation of referral programs as a major gap in knowledge that requires future study.

Second, the intervention might have been more effective in increasing clinicians' use of the 5As if it had included strategies such as booster trainings, reminders or prompts about the ongoing availability of the community exercise program, and additional staff support to help with problem solving (28). Consistent with their patients' aggregate ratings, Group 1 clinicians reported challenges in problem solving with their patients; problem solving is necessary to accomplish Assess and Assist and may be related to a clinician quality that needs further study.

This study has several limitations. Although clinicians were randomized for practical reasons, we had inadequate control group data and therefore used a pre-post analysis. Although the patient sample size was larger than is typically reported for this type of research, patients were nested within a small sample of clinicians from a single geographic location. Participants' awareness of the study topic and objective could have increased the likelihood of patient bias to overreport physical activity counseling. However, the blinding of patients to time point and participation of clinicians in the intervention increased the likelihood that any bias was evenly distributed among time points and clinicians. Finally, by design, this study did not assess patients' behavior change during the study; instead, it aggregated patient ratings nested by clinician. This trade-off was made for practical and logistical reasons because of the focus on an underserved population, but future research would be enhanced by longitudinal assessments of changes in physical activity among patients.

Strengths of the study are that, to our knowledge, this is the first study to assess the effectiveness of a clinician intervention on use of the 5 As for changing physical activity counseling in a medically underserved population. This study is relevant for several reasons. First, it addressed the high prevalence of lifestyle-related chronic conditions in a medically underserved population and the need to eliminate racial/ethnic disparities (20). Second, the intervention was interactive and innovative and had multiple levels. Although it focused on clinicians, it also created tools in the electronic health record for use by the entire clinic, and it initiated a partnership with a community exercise program. Third, this study represents an evaluation of an intervention aimed at translating guidelines into everyday practice, which is not commonly reported in the literature.

This study has 2 main clinical implications. First, clinicians can be taught to improve their physical activity counseling by using the 5 As framework. Second, the intervention can improve confidence in counseling skills among physicians, especially by educating them about community resources for physical activity.

This study also has policy relevance. Primary care is undergoing transformation in the United States. The patientcentered medical home (PCMH) initiative has created a resurgence of interest in helping primary care patients change health behaviors (29) to qualify for higher insurance reimbursements (30). The PCMH standards from the National Committee for Quality Assurance include key ("must-pass") elements for practices to "support self-care" in part by providing educational resources, self-management tools, and counseling to adopt healthy behaviors for least $50 \%$ of patients (31). The changes primary care practices are undertaking are complex, however, and many practices struggle with how to best meet the PCMH standards. This study represents 1 strategy to offer training and tools to help clinicians translate evidence-based guidelines into practice and to address the PCMH-relevant goal of helping patients adopt healthy behaviors.

The results of a clinician-directed intervention designed to increase patient reports of physical activity counseling were mixed. Group 2 clinicians (who took part in the intervention after Group 1) increased their use of the 5As more than 
did Group 1; the difference in use between the 2 groups was due primarily to greater use of Assess and Assist skills among Group 2. Group 2 clinicians improved their awareness of the community exercise program for referral, whereas Group 1 clinicians reported difficulty with problem-solving skills. Future directions are to explore the association between 5 As counseling and patient outcomes such as enrollment and participation in community exercise programs.

\section{Acknowledgments}

We extend our thanks and appreciation for the patients and clinicians who participated in this study. We also thank Carol Moulthroup for her editorial support. Research reported in this publication was supported by the National Cancer Institute of the National Institutes of Health under award no. Ko7CA126985; the Clinicaltrials.gov identifier is NCT01419093. The content is solely the responsibility of the authors and does not necessarily represent the official views of the National Institutes of Health.

\section{Author Information}

Corresponding Author: Jennifer K. Carroll, MD, MPH, University of Rochester Medical Center, Department of Family Medicine, Family Medicine Research Programs, 1381 South Ave, Rochester, NY 14620. Telephone: 585-506-9484 x219. E-mail: Jennifer_carroll@urmc.rochester.edu.

Author Affiliations: Paul C. Winters, Mechelle R. Sanders, Francesca Decker, Thanh Ngo, University of Rochester, Rochester, New York; Christopher N. Sciamanna, Penn State College of Medicine, Hershey, Pennsylvania.

\section{References}

1. Centers for Disease Control and Prevention. National Health and Nutrition Examination Survey NHANES 20122012. http://wwwn.cdc.gov/nchs/nhanes/search/nhanes09_10.aspx. Accessed January 14, 2014.

2. Crespo CJ, Smit E, Andersen RE, Carter-Pokras O, Ainsworth BE. Race/ethnicity, social class and their relation to physical inactivity during leisure time: results from the Third National Health and Nutrition Examination Survey, 1988-1994. Am J Prev Med 2000;18(1):46-53. CrossRef 国 PubMed 圈

3. National Association of Community Health Centers. Community health centers: the return on investments, fact sheet 2012. http://www.nachc.com/client/CHCs\%20ROI\%20final.pdf. Accessed January 14, 2014.

4. Pinto BM, Goldstein MG, Ashba J, Sciamanna CN, Jette A. Randomized controlled trial of physical activity counseling for older primary care patients. Am J Prev Med 2005;29(4):247-55. CrossRef 国 PubMed 国

5. Ackermann RT, Deyo RA, LoGerfo JP. Prompting primary providers to increase community exercise referrals for older adults: a randomized trial. J Am Geriatr Soc 2005;53(2):283-9. CrossRef 圈 PubMed 圈

6. Petrella RJ, Koval JJ, Cunningham DA, Paterson DH. Can primary care doctors prescribe exercise to improve fitness? The Step Test Exercise Prescription (STEP) project. Am J Prev Med 2003;24(4):316-22. CrossRef 圈 PubMed 圈

7. Jay M, Gillespie C, Schlair S, Sherman S, Kalet A. Physicians' use of the 5 As in counseling obese patients: is the quality of counseling associated with patients' motivation and intention to lose weight? BMC Health Serv Res

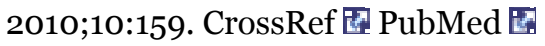

8. Jay M, Schlair S, Caldwell R, Kalet A, Sherman S, Gillespie C. From the patient's perspective: the impact of training on resident physician's obesity counseling. J Gen Intern Med 2010;25(5):415-22. CrossRef 圈 PubMed 圈

9. Harrison RL, Mattson LK, Durbin DM, Fish AF, Bachman JA. Wellness in community living adults: the Weigh to Life program. Patient Educ Couns 2012;86(2):270-6. CrossRef 圈 PubMed 圈

10. Pbert L, Druker S, DiFranza JR, Gorak D, Reed G, Magner R, et al. Effectiveness of a school nurse-delivered

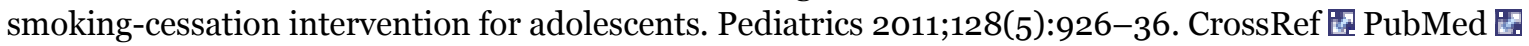

11. Unrod M, Smith M, Spring B, DePue J, Redd W, Winkel G. Randomized controlled trial of a computer-based, tailored intervention to increase smoking cessation counseling by primary care physicians. J Gen Intern Med 2007;22(4):478-84. CrossRef 圈 PubMed 医

12. Glynn TJ, Manley MW. How to help your patients stop smoking: a manual for physicians. NIH Publication 893064 ed. Bethesda (MD): National Cancer Institute; 1989.

13. Whitlock EP, Orleans CT, Pender N, Allan J. Evaluating primary care behavioral counseling interventions: an evidence-based approach. Am J Prev Med 2002;22(4):267-84. CrossRef 国 PubMed 圈

14. Elford RW, MacMillan HL, Wathen CN, Canadian Task Force on Preventive Health Care. Counseling for risky health habits: A conceptual framework for primary care practitioners. Report No. 01-7. London (ON): Canadian Task Force; 2001. 
15. Khan KM, Weiler R, Blair SN. Prescribing exercise in primary care. BMJ 2011;343:d4141. CrossRef 圈 PubMed 圈

16. Swedish National Institute of Public Health. Physical activity in the prevention and treatment of disease 2011. www.fhi.se/PageFiles/10682/Physical-Activity-Prevention-Treatment-Disease-webb.pdf. Accessed January 14, 2014.

17. The Centers for Medicare and Medicaid Services (CMS). Decision memo for intensive behavioral therapy for obesity (CAG-00433N) 2011. http://www.cms.gov/medicare-coverage-database/details/nca-decisionmemo.aspx?\&NcaName=Intensive\%20Behavioral\%20Therapy\%2ofor\% 20Obesity\&bc=ACAAAAAAIAAA\&NCAId=253. Accessed January 14, 2014.

18. Carroll JK, Mohr DC, Spring B, Figueroa-Moseley C, Jean-Pierre P, Sprod LK, et al. What are successful recruitment and retention strategies for underserved populations? Examining physical activity interventions in primary care and community settings. Transl Behav Med 2011;1(2):234-51. CrossRef 国 PubMed 圈

19. Lin JS, O'Connor E, Whitlock EP, Beil TL. Behavioral counseling to promote physical activity and a healthful diet to prevent cardiovascular disease in adults: a systematic review for the U.S. Preventive Services Task Force. Ann Intern Med 2010;153(11):736-50. CrossRef 圈 PubMed 圈

20. Carroll JK, Fiscella K, Epstein RM, Sanders MR, Williams GC. A 5A's communication intervention to promote physical activity in underserved populations. BMC Health Serv Res 2012;12:374. CrossRef 圈 PubMed 圈

21. Epstein RM, Franks P, Fiscella K, Shields CG, Meldrum SC, Kravitz RL, et al. Measuring patient-centered communication in patient-physician consultations: theoretical and practical issues. Soc Sci Med 2005;61(7):151628. CrossRef 圈 PubMed 圈

22. Lewin SA, Skea ZC, Entwistle V, Zworenstein M, Dick J. Interventions for providers to promote a patient-centered approach in clinical consultations. Cochrane Database Syst Rev 2001(4):CDo03267. PubMed

23. Epstein RM. Assessment in medical education. N Engl J Med 2007;356(4):387-96. CrossRef 圈 PubMed 圈

24. Sciamanna CN, Goldstein MG, Marcus BH, Lawrence K, Pinto BM. Accuracy of recall of exercise counseling among primary care patients. Prev Med 2004;39(6):1063-7. CrossRef 国 PubMed 国

25. Cohen J. Statistical power analysis for the behavioral sciences. New York (NY): Academic Press; 1977.

26. Orrow G, Kinmonth AL, Sanderson S, Sutton S. Effectiveness of physical activity promotion based in primary care: systematic review and meta-analysis of randomised controlled trials. BMJ 2012;344:e1389. CrossRef 圈 PubMed 圈

27. Moyer VA. Behavioral counseling interventions to promote a healthful diet and physical activity for cardiovascular disease prevention in adults: U.S. Preventive Services Task Force recommendation statement. Ann Intern Med 2012;157(5):367-71. PubMed 圈

28. Cabana MD, Rand CS, Powe NR, Wu AW, Wilson MH, Abboud PA, et al. Why don't physicians follow clinical practice guidelines? A framework for improvement. JAMA 1999;282(15):1458-65. CrossRef 䍐 PubMed 圈

29. Rosenberg CN, Peele P, Keyser D, McAnallen S, Holder D. Results from a patient-centered medical home pilot at UPMC Health Plan hold lessons for broader adoption of the model. Health Aff (Millwood) 2012;31(11):2423-31. CrossRef圈 PubMed 圈

30. Nocon RS, Sharma R, Birnberg JM, Ngo-Metzger Q, Lee SM, Chin MH. Association between patient-centered medical home rating and operating cost at federally funded health centers. JAMA 2012;308(1):60-6. CrossRef 国 PubMed 圈

31. National Committee for quality Assurance. Patient-centered medical home (PCMH)2011. http://www.ncqa.org/Home/PatientCenteredMedicalHome2011.aspx. Accessed January 14, 2014.

\section{Tables}

Table 1. Sociodemographic and Health Characteristics of Patient Participants $(\mathrm{N}=325)$, Study on Clinician Counseling on Physical Activity, 2009-2011a

\begin{tabular}{|l|r|}
\hline Characteristic & \multicolumn{1}{|c|}{ Value } \\
\hline Sex & $225(70.5)$ \\
\hline Female & $94(29.5)$ \\
\hline Male & \\
\hline
\end{tabular}




\begin{tabular}{|c|c|}
\hline Characteristic & Value \\
\hline \multicolumn{2}{|l|}{ Race/ethnicity } \\
\hline Hispanic & $28(8.8)$ \\
\hline Non-Hispanic white & $53(16.6)$ \\
\hline Non-Hispanic black & $223(69.9)$ \\
\hline Other & $15(4.7)$ \\
\hline \multicolumn{2}{|l|}{ Insurance } \\
\hline Public & $156(61.4)$ \\
\hline Private & $96(37.8)$ \\
\hline None & $2(0.8)$ \\
\hline \multicolumn{2}{|l|}{ Education } \\
\hline Less than high school & $86(27.7)$ \\
\hline High school or equivalent & $100(32.3)$ \\
\hline More than high school & $124(40.0)$ \\
\hline \multicolumn{2}{|l|}{ Employment } \\
\hline Employed & $112(34.8)$ \\
\hline Not employed & $210(65.2)$ \\
\hline \multicolumn{2}{|l|}{ Annual income, $\$$} \\
\hline$<10,000$ & $119(39.8)$ \\
\hline $10,000-20,000$ & $90(30.1)$ \\
\hline$>20,000$ & $49(16.4)$ \\
\hline Chose not to answer & $41(13.7)$ \\
\hline Age, mean (SD), y & $43.2(14.2)$ \\
\hline BMI, mean (SD), kg/m² & $32.3(8.7)$ \\
\hline \multicolumn{2}{|l|}{ Chronic condition } \\
\hline Obesity (BMI $\geq 30$ ) & $174(58.2)$ \\
\hline Hypertension & $157(48.5)$ \\
\hline Chronic pain & $137(42.3)$ \\
\hline Depression & $105(32.4)$ \\
\hline Diabetes & $69(21.3)$ \\
\hline High cholesterol & $78(24.1)$ \\
\hline$\geq 3$ Chronic conditions & $164(51.3)$ \\
\hline
\end{tabular}

Abbreviations: SD, standard deviation; BMI, body mass index.

a Values are numbers and percentages, unless otherwise indicated. Because of missing data, some categories do not sum to 325. Percentages are based on number of participants who responded to a particular question.

Table 2. Overall Physical Activity Exit Interview (PAEI) Scoresa at Baseline, Postintervention,b and 6-Month Follow-Up, Study on Clinician Counseling on Physical Activity, 2009-2011

\begin{tabular}{|c|c|c|c|c|c|c|c|c|c|}
\hline \multirow[b]{2}{*}{ Value } & \multicolumn{3}{|c|}{ Group $1(n=163)$} & \multicolumn{3}{|c|}{ Group $2(n=162)$} & \multicolumn{3}{|c|}{ Both Groups ( $n=319)$} \\
\hline & Baseline & Post & 6 Months & Baseline & Post & 6 Months & Baseline & Post & 6 Months \\
\hline
\end{tabular}




\begin{tabular}{|c|c|c|c|c|c|c|c|c|c|}
\hline \multirow[b]{2}{*}{ Value } & \multicolumn{3}{|c|}{ Group $1(n=163)$} & \multicolumn{3}{|c|}{ Group $2(n=162)$} & \multicolumn{3}{|c|}{ Both Groups $(n=319)$} \\
\hline & Baseline & Post & 6 Months & Baseline & Post & 6 Months & Baseline & Post & 6 Months \\
\hline \begin{tabular}{|l|} 
PAEI \\
Score $(P$ \\
value $) \mathrm{c}$
\end{tabular} & $7.5(-)$ & $\begin{array}{r}8.5 \\
(.27)\end{array}$ & $7.5(.96)$ & $6.3(-)$ & $8.6(.01)$ & $9.1(.006)$ & $6.9(-)$ & $8.6(.01)$ & $8.2(.09)$ \\
\hline $\begin{array}{l}\text { Effect } \\
\text { sized }\end{array}$ & - & $\begin{array}{r}0.21 \\
\text { (Small) }\end{array}$ & $\begin{array}{r}0.49 \\
\text { (Medium) }\end{array}$ & - & $\begin{array}{r}0.46 \\
\text { (Medium) }\end{array}$ & $\begin{array}{r}0.46 \\
\text { (Medium) }\end{array}$ & - & $\begin{array}{r}0.34 \\
\text { (Medium) }\end{array}$ & $\begin{array}{r}0.42 \\
\text { (Medium) }\end{array}$ \\
\hline
\end{tabular}

Abbreviations: -, does not apply.

a The 12-item PAEI (24) was modified for this intervention by adding 3 questions; each question was answered by yes or no, and each yes was counted as 1 point for a possible score range of 0 to 15 points. The survey was administered to 2 groups (Group 1 and Group 2) of patients who were asked to rate their physicians $(n=10)$ on their physical activity counseling.

b Immediately postintervention.

c $P$ values ( $F$ test) determined by comparing postintervention and 6-month follow-up scores with baseline.

d Effect sizes are categorized as small (0.2), medium (0.5), or large (0.8) (25).

Table 3. Physical Activity Exit Interview (PAEI) Scoresa for Each of the 15 Survey Items at Baseline, Postintervention,b and 6-Month Follow-Up, Study on Clinician Counseling on Physical Activity, 2009-2011

\begin{tabular}{|c|c|c|c|c|c|c|}
\hline \multirow[b]{2}{*}{ 5A Question } & \multicolumn{3}{|c|}{ Group $1(n=163), \%$} & \multicolumn{3}{|c|}{ Group 2 (n = 162 ), \% } \\
\hline & Baseline & Post & $\begin{array}{c}6 \\
\text { Months }\end{array}$ & Baseline & Post & $\begin{array}{l}6 \\
\text { Months }\end{array}$ \\
\hline \multicolumn{7}{|l|}{ Ask } \\
\hline Has your provider ever discussed your physical activity? & 84.8 & 96.2 & 83.3 & 81.7 & 81.5 & 87.5 \\
\hline \multicolumn{7}{|l|}{ Advise } \\
\hline $\begin{array}{l}\text { Has your provider ever advised you to become more physically } \\
\text { active? }\end{array}$ & 64.4 & 78.4 & 70.8 & 60.0 & 70.8 & 83.9 \\
\hline $\begin{array}{l}\text { Has your provider ever discussed the reasons you might have } \\
\text { to want to become more physically active? }\end{array}$ & 59.3 & 72.6 & 64.6 & 63.3 & 70.8 & 74.2 \\
\hline \multicolumn{7}{|l|}{ Assess } \\
\hline $\begin{array}{l}\text { Has your provider ever discussed your past experiences with } \\
\text { physical activity? }\end{array}$ & 53.3 & 66.0 & 57.5 & 55.0 & 61.5 & 68.8 \\
\hline $\begin{array}{l}\text { Has your provider ever discussed difficult situations you might } \\
\text { encounter or problems you might have in trying to become } \\
\text { more physically active? }\end{array}$ & 55.9 & 44.9 & 46.8 & 44.1 & 54.7 & 62.5 \\
\hline $\begin{array}{l}\text { Has your provider ever asked you about your willingness to } \\
\text { change your exercise habits?c }\end{array}$ & 51.7 & 58.8 & 54.2 & 45.0 & 63.1 & 65.6 \\
\hline $\begin{array}{l}\text { Has your provider ever asked you about your confidence to } \\
\text { change your exercise habits?c }\end{array}$ & 47.5 & 52.0 & 48.9 & 38.6 & 59.4 & 59.4 \\
\hline \multicolumn{7}{|l|}{ Assist } \\
\hline $\begin{array}{l}\text { Has your provider ever discussed how frequently you should } \\
\text { exercise? }\end{array}$ & 61.7 & 62.0 & 62.5 & 46.7 & 67.7 & 75.0 \\
\hline $\begin{array}{l}\text { Has your provider ever discussed how long you should } \\
\text { exercise? }\end{array}$ & 50.0 & 49.0 & 51.1 & 35.6 & 66.2 & 62.5 \\
\hline $\begin{array}{l}\text { Has your provider ever discussed how hard you should } \\
\text { exercise? }\end{array}$ & 38.3 & 35.3 & 37.5 & 25.0 & 59.4 & 56.3 \\
\hline \multirow[t]{2}{*}{$\begin{array}{l}\text { Has your provider ever discussed the types of exercise you } \\
\text { should do? }\end{array}$} & 55.0 & 64.7 & 56.3 & 45.9 & 58.5 & 62.5 \\
\hline & 18.3 & 19.6 & 20.8 & 10.2 & 21.5 & 20.6 \\
\hline
\end{tabular}




\begin{tabular}{|c|c|c|c|c|c|c|}
\hline \multirow[b]{2}{*}{ 5A Question } & \multicolumn{3}{|c|}{ Group $1(n=163), \%$} & \multicolumn{3}{|c|}{ Group $2(n=162), \%$} \\
\hline & Baseline & Post & $\begin{array}{l}6 \\
\text { Months }\end{array}$ & Baseline & Post & $\begin{array}{l}6 \\
\text { Months }\end{array}$ \\
\hline \multicolumn{7}{|l|}{$\begin{array}{l}\text { Have you and your provider ever put the plan to become more } \\
\text { physically active in writing? }\end{array}$} \\
\hline $\begin{array}{l}\text { Has your provider given you any written materials about } \\
\text { physical activity or exercise during a clinic visit? }\end{array}$ & 39.3 & 35.3 & 31.3 & 22.0 & 33.9 & 36.4 \\
\hline \multicolumn{7}{|l|}{ Arrange } \\
\hline $\begin{array}{l}\text { Has your provider said that he/she is planning to discuss your } \\
\text { physical activity on a future visit? }\end{array}$ & 38.3 & 56.9 & 37.5 & 36.2 & 49.2 & 54.6 \\
\hline $\begin{array}{l}\text { Has your provider ever referred you to other programs, } \\
\text { resources, consultants, etc., to help you with physical activity? } \\
\text { c }\end{array}$ & 38.3 & 47.1 & 41.7 & 39.7 & 47.7 & 40.6 \\
\hline
\end{tabular}

a Scores were converted to percentage of patients answering yes to a given PAEI item. The 12-item PAEI (24) was modified for this intervention by adding 3 questions; each question was answered by yes or no, and each yes was counted as 1 point for a possible score range of 0 to 15 points. The survey was administered to 2 groups (Group 1 and Group 2) of patients who were asked to rate their physicians $(n=10)$ on their physical activity counseling. Generalized estimating equation models controlled for nesting of patients within clinician.

b Immediately postintervention.

c Question added to PAEI for this intervention.

Table 4. Self-Assessed Confidencea of Clinicians $(n=10)$ in Ability to Counsel Patients on Physical Activity at Baseline and 6-Month Follow-Up, Study on Clinician Counseling on Physical Activity, 2009-2011

\begin{tabular}{|c|c|c|c|}
\hline Counseling skill & $\begin{array}{l}\text { Baseline, Mean } \\
\text { (SD) }\end{array}$ & $\begin{array}{l}\text { Follow-up, Mean } \\
\text { (SD) }\end{array}$ & $\begin{array}{c}P \\
\text { Valueb }\end{array}$ \\
\hline Assess exercise history & $3.1(1.0)$ & $4.3(0.6)$ & .003 \\
\hline Adapt counseling to patient situation or needs & $2.8(0.7)$ & $3.8(1.3)$ & .06 \\
\hline Negotiate an exercise plan & $2.8(0.8)$ & $4.0(0.9)$ & .02 \\
\hline Turn setbacks into learning & $2.2(0.7)$ & $3.4(1.7)$ & .04 \\
\hline Help cope with barriers & $2.4(0.9)$ & $4.1(1.9)$ & .02 \\
\hline Counsel in cost effective way & $2.0(0.7)$ & $3.5(1.4)$ & $<.001$ \\
\hline $\begin{array}{l}\text { Knowledge of resources that could meet your patients' } \\
\text { needs }\end{array}$ & $2.1(0.8)$ & $3.5(1.2)$ & .004 \\
\hline Integrate counseling into visit & $3.2(0.7)$ & $4.0(1.1)$ & .04 \\
\hline
\end{tabular}

Abbreviations: SD, standard deviation.

a Based on Likert scale ranging from 1 (not at all confident) to 5 (very confident).

b Paired $t$ test.

The opinions expressed by authors contributing to this journal do not necessarily reflect the opinions of the U.S. Department of Health and Human Services, the Public Health Service, the Centers for Disease Control and Prevention, or the authors' affiliated institutions.

For Questions About This Article Contact pcdeditor@cdc.gov

Page last reviewed: May 29, 2014

Page last updated: May 29, 2014

Content source: National Center for Chronic Disease Prevention and Health Promotion 
Preventing Chronic Disease | Clinician-Targeted Intervention and Patient-Reported Co... Page 11 of 11

Centers for Disease Control and Prevention 1600 Clifton Rd. Atlanta, GA 30333, USA

80o-CDC-INFO (800-232-4636) TTY: (888) 232-6348 - Contact CDC-INFO

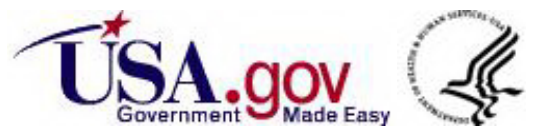

